\title{
3.7 TIMING OF THE PULSAR NP 0527
}

\author{
D. W. RICHARDS* and J. A. ROBERTS** \\ Cornell-Sydney University Astronomy Center, Arecibo Observatory, Arecibo, Puerto Rico
}

\begin{abstract}
Timing measurements have been made of NP 0527 in order to examine its physical relationship with NP 0532. 18 months of timing data have been used to obtain an accurate frequency, rate of change of frequency and position. The calculated 'slowdown age' of the NP 0527 is $3.0 \times 10^{6}$ years which rules out an origin in the supernova explosion of 1054 A.D. The upper limit of its transverse velocity is $0.046 \mathrm{c}$ which is significantly less than the value necessary if it had a common origin with NP 0532.
\end{abstract}

The question of a physical relationship between the pulsar NP 0532 and its neighbor, NP 0527, was first raised by Reifenstein et al. (1969). They suggested that NP 0527 was ejected from the supernova which produced NP 0532 and calculated that if NP 0527 has not been accelerated, it must be moving with a space velocity of $\sim 0.15 \mathrm{c}$. More recently, Gott et al. (1970) have proposed a model wherein both pulsars were initially components of a double star in the nearby association I Geminorum. According to this picture, NP 0527 became a supernova first and both stars 'ran away' from the association, NP 0532 being formed later. Both pulsars were calculated to have space velocities of $\sim 150 \mathrm{~km} / \mathrm{sec}$.

In principle, timing observations of the pulses from NP 0527 can measure the proper motion, and distinguish between these two proposals. Timing observations also provide a measure of $\mathrm{d} f / \mathrm{d} t$, the time rate of change of the pulsar repetition frequency, $f$, and a determination of the position of the pulsar.

We wish to report the preliminary reduction of 18 months of timing observations of NP 0527 made at the Arecibo Observatory. The rather grand scale of the pulses from NP 0527, in comparison with those from NP 0532, is illustrated by the typical pulse shape shown in Figure 1. One component of the double pulse is as wide as the full period of the Crab pulsar. This scale is reflected in the timing accuracies which are reduced by a factor of about 100 , or roughly in the ratio of the width of the first component of NP $0527(30 \mathrm{msec})$ to that of the main pulse in NP $0532(300 \mu \mathrm{sec})$. The errors in the daily mean arrivals (derived as in Richards et al., 1970) are about $1 \mathrm{msec}$ as compared with about $10 \mu \mathrm{sec}$ for NP 0532 .

A set of pulse numbers, $N$, and barycentric arrival times, $t$, was derived for NP 0527 in much the same way as for NP 0532 (Richards et al., 1970; Roberts and Richards, 1970), except that the topocentric arrival times were found by matching a transparent overlay to line-printer plots of the observations, and not by computerized cross correlation. The position and the dispersion constant used for the reductions to the barycenter and to infinite frequency were those given by Zeissig and Richards (1969).

* Present Address: Air Force Cambridge Research Laboratories, Bedford, Mass., U.S.A.

** Permanent Address: CSIRO Radiophysics Laboratory, Epping, N.S.W. 2121, Australia. 
These barycentric arrivals were fitted to a polynomial of the form

$$
N_{\mathrm{fit}}=N_{0}+f_{0} t+\frac{1}{2}(\mathrm{~d} f / \mathrm{d} t)_{0} t^{2},
$$

where $N_{\text {fit }}$ is a continuous variable ( $1 / 2 \pi$ times the pulse phase), $N_{0}$ is a constant, and the subscript 0 denotes values at $t=0$. The residuals from this fit had a quasisinusoidal form with a period of one year, indicating an error in the assumed position.

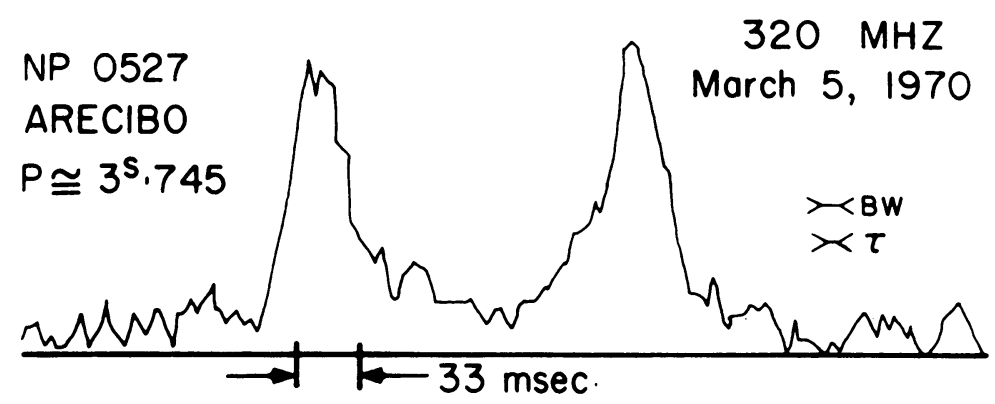

Fig. 1. Typical pulse shape for NP 0527 as obtained at Arecibo.

It turns out that the eccentricity of the earth's orbit is sufficiently great that a revised position could not be found by simply fitting a sinusoid to the residuals, so the following technique was used to determine corrections to the originally assumed right ascension, $\alpha$, and declination, $\delta$.

Let $\hat{\mathbf{s}}$ be the unit vector pointing towards the pulsar, and $\hat{\mathbf{s}}_{0}$ the corresponding unit vector determined by the first estimates of $\alpha$ and $\delta$. The difference, $\Delta \mathbf{s}$, is defined by

$$
\hat{\mathbf{s}}=\hat{\mathbf{s}}_{0}+\Delta \mathbf{s} .
$$

If $\mathbf{r}$ is the vector pointing from the solar system barycenter to terrestrial observing site at the time the pulse arrives, then the barycentric light travel time, $b$, is

$$
\begin{aligned}
b=\mathbf{r} \cdot \hat{\mathbf{s}} & =\mathbf{r} \cdot \hat{\mathbf{s}}_{0}+\mathbf{r} \cdot \Delta \mathbf{s}, \\
& =b_{0}+\Delta b .
\end{aligned}
$$

Here $b_{0}$ is the original estimate of $b$, and the correction $\Delta b$ may be expressed in terms of the corrections $\Delta \alpha, \Delta \delta$ to be made to the right ascension and declination.

Thus $\Delta \mathbf{s}$ is given approximately by

$$
\Delta \mathbf{s}=\frac{\partial}{\partial \alpha}\left(\hat{\mathbf{s}}_{0}\right) \Delta \alpha+\frac{\partial}{\partial \delta}\left(\hat{\mathbf{s}}_{0}\right) \Delta \delta,
$$

or

$$
\begin{aligned}
\Delta \mathbf{s} & =[-\hat{\mathbf{x}} \cos \delta \sin \alpha+\hat{\mathbf{y}} \cos \delta \cos \alpha] \Delta \alpha \\
& +[-\hat{\mathbf{x}} \sin \delta \cos \alpha-\hat{\mathbf{y}} \sin \delta \sin \alpha+\hat{\mathbf{z}} \cos \delta] \Delta \delta,
\end{aligned}
$$

where $\hat{\mathbf{x}}, \hat{\mathbf{y}}, \hat{\mathbf{z}}$ are unit vectors in the Equatorial Cartesian set of coordinates (at epoch 1950.0). Writing

$$
\mathbf{r}=\hat{\mathbf{x}} r_{x}+\hat{\mathbf{y}} r_{y}+\hat{\mathbf{z}} r_{z}
$$


we find

where

$$
\Delta b=\Delta \alpha R_{\alpha}+\Delta \delta R_{\delta}
$$

$$
\begin{aligned}
& R_{\alpha}=-r_{x} \cos \delta \sin \alpha+r_{y} \cos \delta \cos \alpha, \\
& R_{\delta}=-r_{x} \sin \delta \cos \alpha-r_{y} \sin \delta \sin \alpha+r_{z} \cos \delta
\end{aligned}
$$

The functions $R_{\alpha}$ and $R_{\delta}$ can be computed when $b_{0}$ is calculated, and used later in a least squares solution for $\Delta \alpha$ and $\Delta \delta$. A fit to the function formed from the sum of the r.h.s. of equation (1) and $f_{0}$ times the r.h.s. of equation (2) yielded the residuals shown in Figure 2. The fitted frequency and rate of change, referred to epoch Julian Date 2440400.5 are:

$$
\begin{aligned}
f & =0.26698766361 \pm\left(87 \times 10^{-11}\right) \mathrm{Hz} \text { UTC, } \\
\mathrm{d} f & =(-2.8481 \pm 0.0041) \times 10^{-15} \mathrm{sec}^{-2}
\end{aligned}
$$

The 'age' of the pulsar, $-f /(\mathrm{d} f / \mathrm{d} t)$, is $3.0 \times 10^{6} \mathrm{yr}$. This appears to rule out an origin in the supernova explosion of $1054 \mathrm{AD}$ as suggested by Reifenstein et al., independently of any consideration of the proper motion. The new right ascension and declination are:

$$
\begin{aligned}
& \alpha(1950.0)=05^{\mathrm{h}} 25^{\mathrm{m}} 52^{\mathrm{s}} .08 \pm 0.07 \\
& \delta(1950.0)=21^{\circ} 56^{\prime} 32^{\prime \prime} \pm 16^{\prime \prime}
\end{aligned}
$$

Because the pulsar lies almost in the ecliptic plane, and near the summer solstice, the declination is poorly determined. The formal one standard deviation errors from the least squares fit quoted above should be considered optimistic since there may be systematic errors. In this preliminary reduction no allowance was made for the different receiver time constants and bandwidths used on different days, and an old value of the dispersion constant, $B$, was used. To keep the effect of possible errors in $B$ at a minimum, only observations at $318 \mathrm{MHz}$ or higher have been used in this reduction. Possible time variation of $B$ has been neglected.

Any proper motion of the source would produce quasi-sinusoidal residuals with a period of one year and an amplitude that increased linearly with the time. The fitting procedure determines the source position near the mid-point of the data span, so that proper motion residuals in Figure 2 would be zero near the mid-point and form a one-year sinusoid with amplitude increasing away from the mid-point, and a phase reversal at the mid-point. No such trend is evident, and to be within the errors the amplitude must be less than $\pm 8 \mathrm{msec}$ after 9 months. For a distance of $2 \mathrm{kpc}$ this gives an upper limit of $0.2 \mathrm{c}$ for any transverse velocity parallel to the ecliptic plane.

A much more stringent limit on the proper motion may be deduced from the measured value of $\mathrm{d} f / \mathrm{d} t$, as Staelin (1970) has pointed out. Qualitatively Staelin's argument is the following. If the pulsar has a constant velocity with a component transverse to the line of sight, then the distance to the pulsar will be increasing in a 
non-linear fashion. This leads to a changing Doppler effect and hence a decrease of the apparent pulsar repetition frequency. Shklovsky (1969) and Detre (1969) have in fact suggested that this effect could account for all the observed frequency change for pulsars with periods $\sim 1 \mathrm{sec}$. Here we place an upper limit on any transverse velocity by assuming all the measured $\mathrm{d} f / \mathrm{d} t$ is due to the motion of the pulsar.

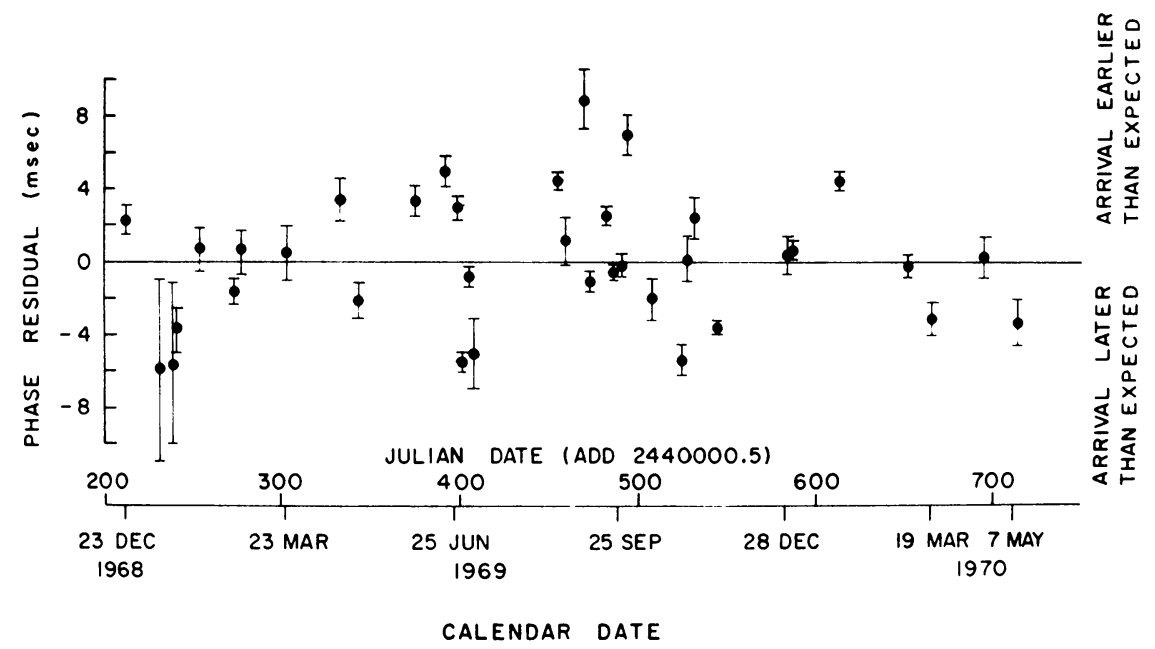

Fig. 2. Phase residual for simultaneous quadratic and position correction fit. Some of the scatter is due to systematic effects (see text).

Resolve the (constant) velocity $\mathbf{v}$ of NP 0527 into components perpendicular $\left(v_{p} \hat{\mathbf{p}}\right)$ and parallel $\left(v_{s} \hat{\mathbf{s}}\right)$ to the line of sight. As before, $\hat{\mathbf{s}}$ points from the solar system barycenter to the pulsar. If $f_{0}$ is the (constant) pulsation frequency of the pulsar in its rest frame, then we observe a Doppler shifted frequency

Thus

$$
f=f_{0}(1-\mathbf{v} \cdot \hat{\mathbf{s}} / c) \text {. }
$$

or

$$
\mathrm{d} f / \mathrm{d} t=-\left(f_{0} / c\right) \mathbf{v} \cdot d \hat{\mathbf{s}} / d t
$$

$$
\begin{aligned}
\mathrm{d} f / \mathrm{d} t & \approx-\left(f_{0} / c\right) \mathbf{v} \cdot \hat{\mathbf{p}}\left(v_{p} / r\right) \\
& =-\left(f_{0} v_{p}^{2}\right) / c r
\end{aligned}
$$

where $r$ is the distance to the pulsar. Hence

$$
v_{p}=\left[-\left(r c / f_{0}\right) \mathrm{d} f / \mathrm{d} t\right]^{1 / 2} .
$$

Using $r=2 \mathrm{kpc}$ and $f_{0}$ and $\mathrm{d} f / \mathrm{d} t$ from the fit discussed above, we find

$$
v_{p} \approx 13800 \mathrm{~km} / \mathrm{sec}=0.046 c .
$$


The corresponding upper limit on proper motion is 1 ".4 arc/yr. This upper limit definitely eliminates the Reifenstein et al. model, but the limit is far above the values derived by Gott et al.

For their model Gott et al. calculated that $\mathrm{d} f / \mathrm{d} t$ due only to pulsar spin down would be $(0.64-1.43) \times 10^{-15} \mathrm{sec}^{-2} \pm 15 \%$. The contribution to $\mathrm{d} f / \mathrm{d} t$ from their predicted transverse velocity is quite small and hence their estimate of $\mathrm{d} f / \mathrm{d} t$ falls short of the observed value by at least a factor of 2 .

If NP 0527 is a neutron star of one solar mass, radius $10 \mathrm{~km}$, and uniform density, the observed $\mathrm{d} f / \mathrm{d} t$ corresponds to an energy loss rate of $3.6 \times 10^{31} \mathrm{erg} / \mathrm{sec}$. The radio energy density of about $5 \times 10^{-27} \mathrm{~J} \mathrm{~m}^{-2} \mathrm{~Hz}^{-1} /$ pulse (Zeissig and Richards, 1969) can account for only $\sim 6 \times 10^{29} \mathrm{erg} / \mathrm{sec}$, assuming a bandwidth of $1000 \mathrm{MHz}$, a distance of $2 \mathrm{kpc}$, and a beam solid angle of $4 \pi / 50$ sterad. If we suppose that nearly all of the $3 \times 10^{31} \mathrm{erg} / \mathrm{sec}$ is radiated in the optical, the absolute magnitude of the pulsar will be about +10 . At $2 \mathrm{kpc}$, neglecting absorption, it will be fainter than magnitude +21 , near the borderline of detectability in previous searches (Kristian, 1970; Willstrop, 1970).

\section{Acknowledgements}

The assistance of G. A. Zeissig in securing the observations is greatly appreciated. We also thank the MIT Haystack Observatory for provision of computer time; the staff of the Arecibo Observatory for assistance in all phases of the work; and M. E. Ash, I. I. Shapiro, and W. B. Smith of the MIT Lincoln Laboratory for ephemerides. Additional support under contract F-44620-69-C-0092 of the U.S. Air Force Office of Scientific Research is gratefully acknowledged. The Arecibo Observatory is operated by Cornell University under contract with the National Science Foundation, and with partial support from the Advanced Research Projects Agency.

\section{References}

Detre, L.: 1969, IAU Commission 27 Information Bulletin on Variable Stars No. 380.

Gott, J. R., Gunn, J. E., and Ostriker, J. P.: 1970, Astrophys. J. 160, L91.

Kristian, J.: 1970, this symposium, Paper 2.3, p. 87.

Reifenstein III, E. C., Brundage, W. D., and Staelin, D. H.: 1969, Phys. Rev. Letters 22, 311.

Richards, D. W., Pettengill, G. H., Counselman III, C. C., and Rankin, J. M.: 1970, Astrophys. J. 160, L1.

Roberts, J. A. and Richards, D. W.: 1970, this symposium, Paper 2.11, p. 125.

Shklovsky, I. S.: 1969, Astron. Zh. 46, 715, translated in Soviet Astron. 13, 562 (1970).

Staelin, D. H.: 1970, personal communication.

Willstrop, R. V.: 1970, this symposium, Paper 3.9, p. 222.

Zeissig, G. A. and Richards, D. W.: 1969, Nature 222, 150.

\section{Discussion}

J. G. Davies: To add to the confusion of this subject it is worth noting that the pulsar with the second longest known period (JP 2319) is $1 \frac{1}{2}^{\circ}$ from Cas A. If it were ejected from the supernova, it will have to be travelling near the speed of light and, further, its dispersion measure makes it rather unlikely that it is at the same distance as the radio source. 
D. Staelin: Dr. Sutton and I wish to note that the suggestion of Reifenstein, Brundage, and Staelin (1969) that NP 0527 may have been ejected from the Crab Nebula can now be ruled out by the slowdown rate reported here by Richards. If NP 0527 had indeed been ejected from the Crab about 1000 years ago, then the transverse velocity of NP 0527 would be greater than $0.1 \mathrm{c}$. The resulting second-order Doppler effect would produce an apparent rate of change in period more than twice the measured value, and hence NP 0527 must have a different origin. 